\title{
Intra-Articular Glomus Tumor- A Rare Presentation
}

To the Editor,

Glomus tumors are rare, benign, vascular painful swellings arising from glomus bodies. They represent less than 1.6\% of all benign soft tissue neoplasms (1), are usually found between ages 30 and 50 years and are more common in women than men with a ratio of 2:1 (2). They are commonly seen in the subungual region of digits but ectopic locations are not uncommon (3). Other rare locations include the extremities, trunk, and even the viscera. The forearm is the most common extra digital location and the shoulder and back the least common sites of involvement by this tumor. In the shoulder area, glomus tumor has been reported to arise from bone (scapula), muscle (deltoid muscle) and soft tissue (4).

A 38-year-old lady presented with gradually progressive, non-radiating pain in the right shoulder with stiffness for 10 years. The pain was aggravated on movement and relieved on rest with no past history of trauma/swelling. The routine blood tests were normal. Magnetic Resonance Imaging (MRI) with contrast revealed an intensely-enhancing welldefined loculated intra-articular mass in the right shoulder joint without calcification that was causing scalloping in the humeral head without any marrow edema (Figure 1A,B). The differential diagnosis considered was a synovial sarcoma or an intra-articular giant cell tumor of the tendon sheath. Surgical excision of the tumor was performed and the tumor pieces were curetted and removed. The tumor pieces altogether measured approximately $3 \mathrm{~cm}$ in diameter. Histopathology revealed a variegated lesion, with sheets of small bland monomorphic cells having a well-defined cytoplasm interspersed by variable calibre thin walled blood vessels, some with branching, staghorn pattern (Figure 1C-E). Immunohistochemistry showed these cells to be positive for vimentin (Figure 1F), smooth muscle actin (SMA) (Figure 1G), and caldesmon (Figure 1H) but negative for synaptophysin (Figure 1I), CD31 (Figure 1J) and S-100 (Figure 1K). The histopathology and immunohistochemical profile of the tumor were consistent with an intra-articular glomus tumor. The patient's pain and stiffness resolved completely after surgical removal of the mass. The patient was lost for follow-up.
These rare slow-growing pericytic tumors are seen commonly in the distal extremities. Glomus tumor presenting intraosseously is rare and only 22 cases of primary intraosseous glomus tumor have been reported in the world literature (4). Recurrence is noted due to inadequate excision. The diagnosis of glomus tumor should be kept in mind in cases with chronic shoulder pain with swelling.

\section{REFERENCES}

1. Soule EH, Ghormley RK, Bulbulian AH. Scientific exhibits: Primary tumors of the soft tissues of the extremities exclusive of epithelial tumors: An analysis of five hundred consecutive cases. AMA Arch Surg. 1955;70:462-74.

2. Diao E, Moy OJ. Common tumors. Orthop Clin North Am. 1992;23:187-96.

3. McDermott EM, Weiss AP. Glomus tumors. J Hand Surg Am. 2006;31:1397-400

4. Gautam VK, Agarwal PK, Maini L, Prakash A. Intraosseous glomus tumor in acromion process of scapula. Orthopedics. 2008;31:406.

\section{Ritesh SACHDEV}

Department of Pathology, Lab Medicine and Transfusion Medicine, Medanta - The Medicity Hospital, Sector 38, GURGAON, INDIA

E-mail: sachdev05@gmail.com

Phone: + 09013590875

Ishani MOHAPATRA

Department of Pathology, Lab Medicine and Transfusion Medicine, Medanta - The Medicity Hospital, Sector 38, GURGAON, INDIA

\section{Shalini GOEL}

Department of Pathology, Lab Medicine and Transfusion Medicine, Medanta - The Medicity Hospital, Sector 38, GURGAON, INDIA

\section{Smeeta GAJENDRA}

Department of Pathology, Lab Medicine and Transfusion Medicine, Medanta - The Medicity Hospital, Sector 38, GURGAON, INDIA 

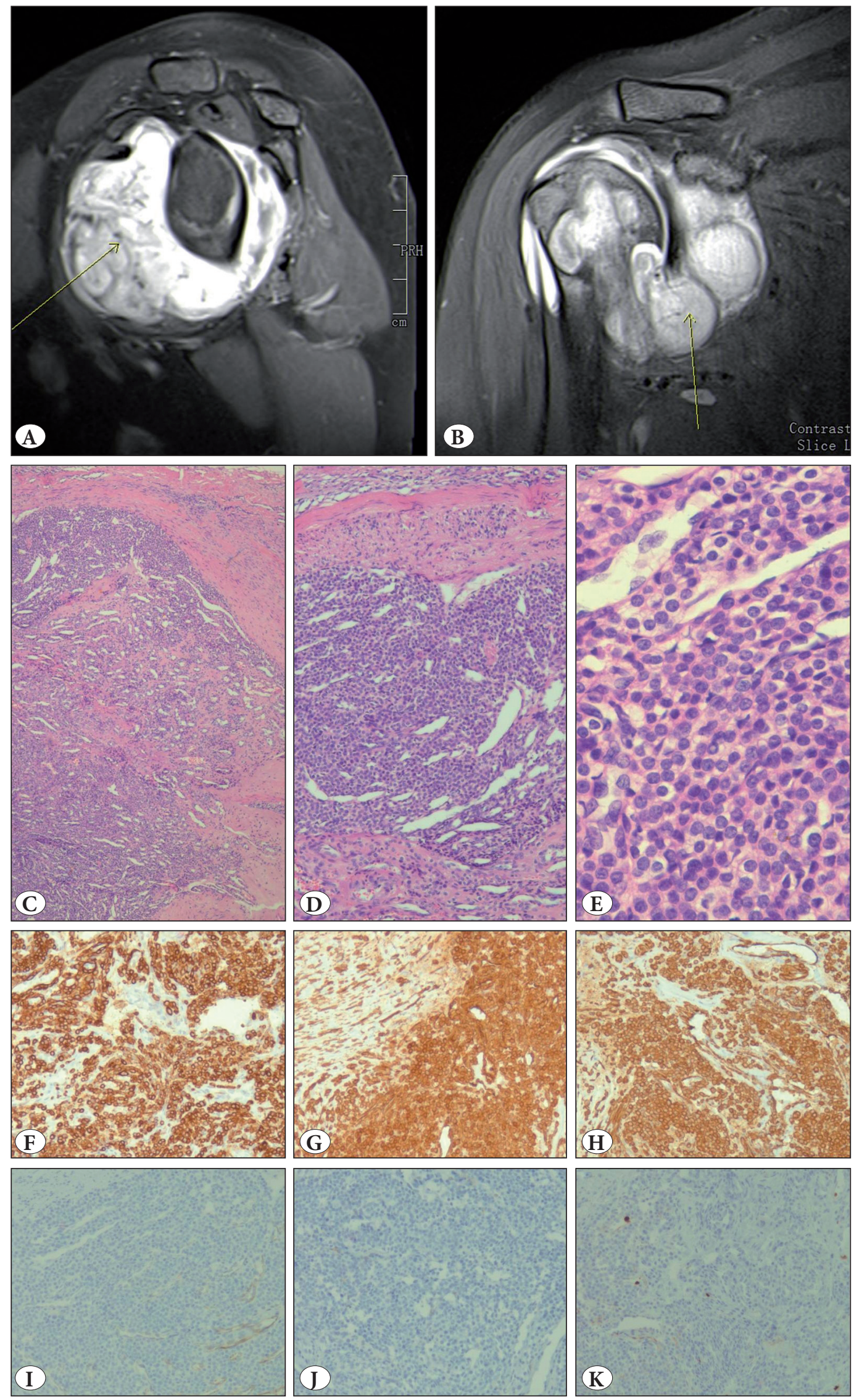
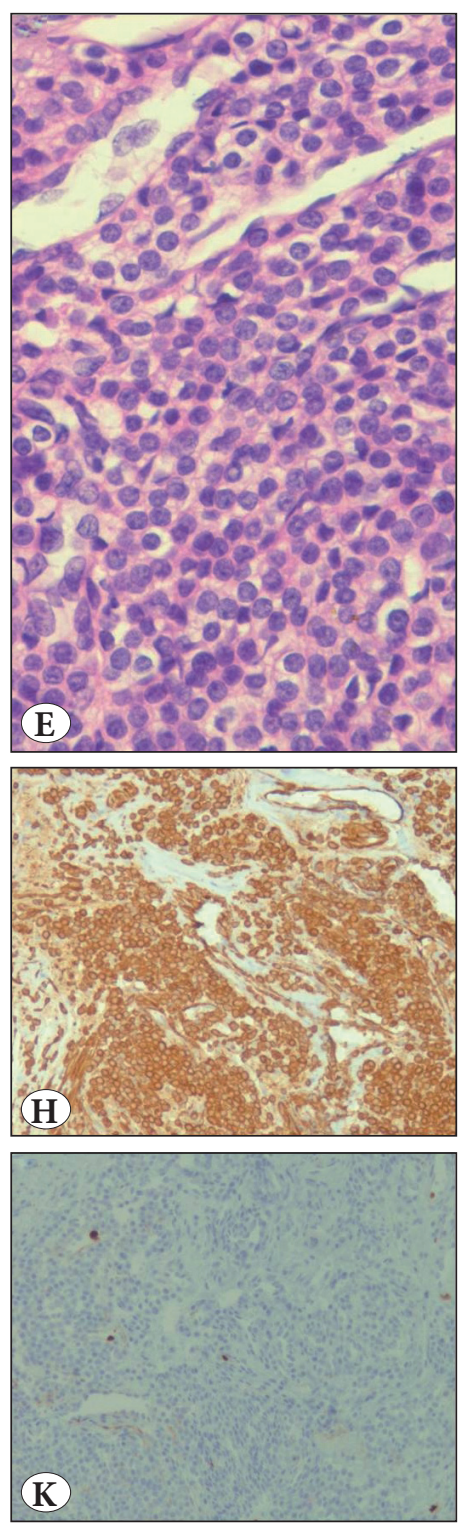

Figure 1: A,B) MRI

showing intensely-enhancing well-defined loculated intra-articular mass in the right shoulder joint without calcification causing scalloping in the humeral head without any marrow edema. C-E) Histopathology revealed sheets of small bland monomorphic cells having well-defined cytoplasm interspersed by variable calibre thin walled blood vessels, some with branching, staghorn pattern (H\&E; x100, x200, x400). F) Vimentin positivity in tumor cells (vimentin; x100). G) SMA positivity in tumor cells (SMA; $x 100)$. H) Caldesmon positivity in tumor cells (Caldesmon; $\mathrm{x} 100)$. I) Tumor cells are negative for synaptophysin (synaptophysin; x100). J) Tumor cells are negative for CD31 (CD31; x100) and K) Tumor cells are negative for S-100 (S-100; x100). 